\title{
O PEDIR AJUDA: CONCEPÇÕES DOS ESTUDANTES DO ENSINO FUNDAMENTAL
}

\author{
Tania Maria Serafim
}

Pedagoga e mestre em Psicologia, Desenvolvimento Humano e Educação pela Faculdade de Educação da Unicamp. Professora efetiva da rede estadual de ensino.

\begin{abstract}
Evely Boruchovitch
Psicóloga pela Universidade do Estado do Rio de Janeiro, Ph.D em Educação pela University of Southern California, Los Angeles, Professora Livre Docente do Departamento de Psicologia Educacional da Faculdade de Educação da Universidade Estadual de Campinas-Unicamp, Membro do Grupo de Estudos e Pesquisas em Psicopedagogia (Gepesp) da Unicamp. Pesquisadora do CNPq.
\end{abstract}

\section{Resumo}

Reconhecendo a carência de pesquisas nacionais acerca da estratégia de pedir ajuda, o presente estudo objetivou conhecer o uso e a concepção de pedir ajuda em 60 estudantes de ambos os sexos de $2^{a}$ a $4^{a}$ série do ensino fundamental. Os instrumentos de coleta de dados foram uma prancha contendo uma situação problema hipotética e uma entrevista estruturada desenvolvidos para o presente estudo. Os dados foram examinados qualitativamente por meio da análise de conteúdo. Resultados indicam que a estratégia de pedir ajuda é bastante relatada pelos participantes para a realização de atividades acadêmicas ou cotidianas. $O$ pedido de ajuda é expresso verbalmente. Os familiares são as principais pessoas a quem os estudantes recorrem no momento de dificuldade. Os dados são discutidos em termos de reconhecer a importância da estratégia de pedir ajuda e da necessidade de novos estudos que aprofundem o conhecimento acerca dessa estratégia.

Palavras-chave: psicologia cognitiva, estratégia de pedir ajuda, estratégia de aprendizagem.

\section{BASIC EDUCATION STUDENTS`CONCEPTIONS OF HELP SEEKING}

\begin{abstract}
The national research about help seeking strategy is slim. In this sense, this study investigated the conceptions about help seeking of 60 students of both sexes from $2^{\text {nd }}$ to $4^{\text {th }}$ grade education. Data collection instruments were both a problem-solving hypothetical situation related to help seeking and a structured interview specially developed for this study. The data were examined qualitatively by means of content analysis. Results indicated that help seeking strategy is frequently reported by students to do their academic activities or daily. The request for assistance is expressed verbally. Family members ate the key persons to whom the students seek at the time of difficulty. Data are discussed not only in terms of the need of acknowledging the importance of help seeking strategy, but also in terms of the need to deepen the knowledge about this strategy.

Keywords: cognitive psychology, help seeking strategy, learning strategy.
\end{abstract}

\section{LO PEDIR AYUDA: CONCEPCIONES DE LOS ESTUDIANTES DE LA EDUCACIÓN BÁSICA}

\section{Resumen}

Reconociendo la falta de investigación acerca de la estrategia nacional para pedir ayuda, este estudio investigó el uso y la concepción de la estrategia para pedir la 
ayuda de 60 estudiantes de ambos sexos de $2^{\circ}$ a $4^{\circ}$ grado de educación. Los instrumentos de recolección de datos fueron "Medios para la evaluación del diseño de los Estudiantes de la solicitud de ayuda" y "Entrevista - entender el diseño de los Estudiantes de la solicitud de ayuda". Los datos se analizaron cualitativamente mediante análisis de contenido. Los resultados indican que la estrategia de pedir ayuda es a menudo utilizada para actividades académicas o diario. La solicitud de asistencia se expresa verbalmente. Miembros de la familia son las personas clave que los alumnos utilizan en el momento de dificultad. Los datos se analizan en términos de reconocer la importancia de la estrategia para pedir ayuda y la necesidad de más estudios para profundizar aún más el conocimiento sobre esta estrategia.

Palabras clave: psicología cognitiva, estrategia para la ayuda, estrategia de aprendizaje.

\section{INTRODUÇÃO}

Existem opiniões distintas sobre a utilidade da estratégia de pedir ajuda para aprendizagem. Primeiramente, pedir ajuda era visto como um padrão maladaptativo de comportamento que poderia provavelmente interferir no desenvolvimento da auto-confiança e competência do estudante. Contudo, no início da década de 80 do século passado uma nova visão sobre pedir ajuda emergiu, com base na perspectiva da aprendizagem auto-regulada. O pedir ajuda, nessa perspectiva passou a ser visto como uma estratégia adaptativa que o estudante pode utilizar quando se depara com dificuldades que sozinho não consegue resolver (Karabenick, 1998; Newman, 1991; 1994; Ryan \& Pintrich, 1997).

A perspectiva da aprendizagem auto-regulada rompe com a idéia de pedir ajuda como um padrão de dependência, considerando-a como uma estratégia legítima para lidar com desafios, e situações de dificuldade e evidenciando o papel dos recursos internos para determinar quando a ajuda é necessária. Além disso, reconhece a importância dos pares e dos fatores motivacionais na ação de pedir ajuda por parte da criança quando necessário. (Marchand \& Skinner, 2007).

A literatura internacional apresenta diversas pesquisas e publicações acerca da estratégia de pedir ajuda, as quais, presumivelmente, se iniciaram na década de 80 do século passado. Os estudos tratam da utilização dessa estratégia, por parte de alunos, desde o início da escolarização até o contexto universitário (Karabenick \& Knapp, 1991; Newman, 1990, 1991; Van der Meij, 1988, 1990).

A estratégia de pedir ajuda se caracteriza por ser tanto uma estratégia metacognitiva quanto de aprendizagem auto-regulada. Alunos auto-regulados são metacognitivos, intrinsecamente motivados e envolvidos em sua própria 
aprendizagem. Uma característica específica de estudantes auto-regulados é a habilidade de se beneficiarem de outras pessoas, como um recurso para lidar com dificuldades no seu processo de aprendizagem. Pedir ajuda requer consciência de que se necessita dela, portanto, envolve a capacidade metacognitiva. Inclui também a decisão de solicitar ou não ajuda, que diz respeito à motivação, além da ação ou do aspecto comportamental que envolve mobilizar estratégias para que outras pessoas se disponham a ajudá-lo.

De acordo com Newman (2002), os alunos que monitoram seu próprio desempenho escolar, mostram consciência de suas dificuldades e buscam superá-las pedindo ajuda a alguém estão demonstrando um comportamento maduro e estratégico. Ao pedir ajuda, o estudante pode superar uma possível falha, manter o envolvimento na tarefa, completar a tarefa com sucesso e aumentar a autonomia em relação à própria aprendizagem. Newman (1994) menciona que a decisão de pedir ajuda é tomada por um sistema afetivomotivacional (system motivational-affective). Relata este autor que o pedido de ajuda adaptativo é definido por uma seqüência de decisões e ações que o aluno deve tomar. Ressalta que pedir ajuda adaptativo requer competência cognitiva, competência social, recursos pessoais e motivacionais (Newman, 1998, 2000). A competência cognitiva envolve o monitoramento da própria aprendizagem, o que implica em o aluno saber quando a ajuda é necessária e como formular a questão que trará a informação de que precisa ("É necessário que eu peça ajuda?"; "Como eu devo formular a questão?"). Por sua vez, a competência social refere-se a reconhecer que outras pessoas podem ajudar e saber distinguir quem é a melhor pessoa ("A quem eu devo perguntar? Professor ou colega?"). Além disso, envolve também saber realizar o pedido por ajuda de forma socialmente apropriada. Já os recursos afetivo-motivacionais são relativos às percepções de competência e de auto-eficácia, às expectativas para o sucesso e às atribuições de causalidade do estudante. Esses recursos internos lhe permitem admitir suas limitações e tentar superá-las, ao pedir assistência (Newman, 1994, 1998). Por último, é imprescindível que o estudante manipule e elabore a ajuda que recebeu.

Dentre as várias estratégias de aprendizagem utilizadas pelos alunos brasileiros destaca-se o pedir ajuda, encontrada em estudos nacionais desenvolvidos por Boruchovitch (1995, 1998, 1999, 2006); Costa (2000); Schilieper, (2001) e Serafim (2004). Os dados revelaram, de modo geral, que os 
estudantes, ao se depararem com tarefas difíceis, relatam que costumam pedir ajuda do professor ou dos colegas. Tendo em vista a importância dessa estratégia para a aprendizagem e os poucos estudos voltados para investigá-la melhor, em nosso meio, a presente pesquisa visa descrever a concepção dos estudantes acerca da estratégia Pedir Ajuda, tendo como referencial teórico a perspectiva da aprendizagem auto-regulada, na qual o estudante pode ser caracterizado pelo seu controle sobre os resultados acadêmicos, bem como pelo seu papel ativo no processo de aprendizagem.

\section{MÉTODO}

\section{Participantes}

A amostra contou com a participação de 60 estudantes, com idade de 7 a 10 anos, de ambos os sexos que após autorização dos responsáveis foram aleatoriamente selecionados por meio de sorteio de uma amostra maior de 159 estudantes. Mais precisamente, compuseram a pesquisa 20 estudantes da $2^{a}$ série, 20 da $3^{a}$ e 20 da $4^{a}$ série escolar. Entre os participantes $50 \%$ era do sexo masculino e $50 \%$ do sexo feminino. A média de idade foi de 9 anos.

\section{Instrumento}

Os dados relativos à concepção dos estudantes acerca da estratégia de pedir foram coletados por meio da "Prancha Para Avaliação da Concepção do Pedir Ajuda de Alunos" e da "Entrevista - Conhecendo a Concepção de Pedir Ajuda de Alunos". A Prancha para Avaliação da Concepção do Pedir Ajuda foi desenvolvida por Serafim e Boruchovitch (2007), a partir da literatura da área para motivar o participante a responder sobre sua concepção de pedir ajuda. Consta de uma história ilustrada que contém uma situação-problema que visa suscitar o uso da estratégia de pedir ajuda.

A situação-problema proposta foi a seguinte: "Ana e Pedro vão viajar de férias com seus pais para a casa de seus avôs. Eles organizaram uma mala cheia de seus brinquedos favoritos para levar. A mala ficou muito pesada. Agora eles não conseguem carregá-la. Se você estivesse na situação deles, o que você faria para colocar essa mala no carro, com todos os brinquedos que eles tanto gostam?". Após a apresentação da situação-problema, os participantes responderam à entrevista estruturada para se identificar a concepção deles acerca do pedir ajuda de autoria de Serafim et al (2007). É composta de 11 
questões abertas. Um exemplo de questão da entrevista, a título de ilustração, é o que se segue: "O que é pedir ajuda, para você?"

\section{Procedimento de coleta de dados}

Depois de o projeto ter sido aprovado pelo Comitê de Ética (processo no 754) foi realizado um contato com a escola no qual foram esclarecidos os objetivos da pesquisa, bem como sua importância para a produção e o avanço do conhecimento acadêmico e do pedagógico. Foi garantido à direção da escola que esta não teria seu nome mencionado, que a coleta de dados com alunos e professores seria realizada nos horários de maior conveniência da instituição. Foi enviado aos pais dos estudantes um termo de consentimento esclarecendo a sua forma de participação.

Após estabelecer um bom rapport com os participantes, o instrumento foi administrado, individualmente, pela primeira autora somente nos alunos sorteados cujos pais autorizaram a participação. Ela fez a leitura em voz alta de todas as questões e anotou na íntegra as repostas dos participantes. A coleta de dados teve a duração média de 30 minutos, variando em função das séries e idades dos participantes.

\section{Procedimento de análise de dados}

As respostas dos participantes acerca da estratégia de pedir ajuda foram estudadas por análise de conteúdo (Bardin, 1991) utilizando um sistema de categorização de resposta para cada questão. Conforme descrito por Boruchovitch (1993), cada categoria foi definida operacionalmente a fim de aumentar a consistência da análise de dados e um conjunto de regras para a classificação das repostas foi cuidadosamente desenvolvido por Serafim et al (2007). Cabe destacar que durante o processo de categorização, dois juízes independentes foram escolhidos, com a finalidade de classificar as respostas dos participantes de acordo com as categorias estabelecidas para cada questão. Cada juiz analisou 12 repostas dadas por diferentes participantes que foram selecionadas aleatoriamente. A porcentagem de correspondência obtida na "Prancha Para Avaliação da Concepção do Pedir Ajuda de Alunos" foi de $100 \%$ e na "Entrevista - Conhecendo a Concepção do Pedir Ajuda de Alunos" variou de 90 a $80 \%$. É importante ressaltar que, como parte do processo de análise, os 
cálculos foram baseados no número de repostas dadas pelos participantes e não na quantidade de participantes.

\section{RESULTADOS}

As respostas dos alunos à situação-problema, examinadas pela análise de conteúdo. Emergiram três categorias de repostas, "Procurar Assistência Social" $(N=37 ; 61,7 \%)$ e "Outra Solução" ( $N=20 ; 33,3 \%)$ e "Não Sei" ( $N=3 ; 5 \%)$. Conforme pode ser observado, a estratégia de pedir ajuda foi mencionada pela maior parte da amostra, sugerindo que essa estratégia é está presente no repertório de estratégias de aprendizagem.

A primeira questão da entrevista buscava conhecer a concepção de pedir ajuda dos participantes e dizia "O que é pedir ajuda?". "Impossibilidade de Realização Independente" ( $N=33 ; 55 \%)$, "Busca de Apoio" ( $N=14 ; 23,3 \%)$ e "Não Sei" ( $N=13 ; 21,7 \%)$ foram as principais categorias que surgiram. A maior parte da amostra associou a estratégia de pedir ajuda com a dificuldade ou falta de conhecimento para resolver sozinho alguma situação.

Conhecer a percepção do estudante acerca da criança que pede ajuda era a finalidade da questão 2 que indagava "Para você, como é uma criança que pede ajuda?" Surgiram quatro categorias de respostas: "Atributos Gerais" ( $N=26$; 43,3\%); Impossibilidade de Realização Independente" ( $N=11 ; 18,3 \%)$; "Pedido Verbal e Comportamento Valorizado" ( $N=7 ; 11,7 \%)$ e "Não Sei" ( $N=16 ; 26,7 \%)$. Foi interessante notar que, a criança que pede ajuda, foi definida pela maior parte dos alunos pelas características gerais do ser humano. É interessante notar que um valor expressivo de estudantes não soube atribuir características a uma criança que pede ajuda.

A questão 3 tinha por objetivo saber se o participante utilizava a estratégia de pedir ajuda e conhecer sua justificativa para o seu uso ou não. "Você pede ajuda? Porque?" Foram encontradas três categorias de repostas: "Impossibilidade de Realização Independente" ( $\mathrm{N}=52 ; 88,1 \%)$; "Prazer de Pedir Ajuda" ( $N=1 ; 1,7 \%)$ e "Não Sei" ( $N=6 ; 10,2 \%)$. Do total de participantes, apenas um estudante da $3^{a}$ série afirmou que não pedia ajuda. Pode-se constatar que a dificuldade para enfrentarem sozinhos uma situação adversa foi a principal explicação dada pela maior parte da amostra para o uso do pedir ajuda. Foi possível ainda observar que, apesar de os participantes afirmaram 
utilizar a estratégia de pedir ajuda, alguns desconhecem ou não sabem explicar o motivo pelo qual a solicitam.

Investigar em quais circunstâncias o estudante pede ajuda foi o tema tratado na questão 4 que perguntava "Em que situações você pede ajuda?" A análise de conteúdo apontou quatro categorias de repostas: "Situações Escolares" ( $\mathrm{N}=30 ; 44,1 \%)$; "Impossibilidade de Realização Independente" $(N=26 ; 38,3 \%)$; "Momentos Difíceis" $(N=6 ; 8,8 \%)$ e "Não Sei" ( $N=6 ; 8,8 \%)$. A maioria das respostas dos participantes referia-se a pedir ajuda para a realização das atividades escolares. Pedir ajuda nos momentos de dificuldade para realizar uma ação de forma autônoma ocorreu em menor proporção.

A questão 5 visava conhecer a forma que estudante utiliza para pedir ajuda. A pergunta feita foi "Como é que você pede?". Surgiram três categorias de respostas: "Pedido Oral Simples" ( $N=31 ; 52,5 \%)$; "Pedido Oral com Ênfase na Adequação Social" ( $N=25 ; 42,4 \%)$ e "Não Sei" ( $N=3 ; 5,1 \%)$. A maior parte da amostra indicou que o pedido de ajuda ocorre verbalmente, isto é, ou o aluno chama uma pessoa ou vai até ela e manifesta o seu pedido. Outros participantes ressaltaram o uso de formas socialmente valorizadas, como por favor e obrigado, para a realização do pedido de ajuda.

A quem os participante pedem ajuda foi o tema abordado na questão 6 . A questão proposta foi: "Para quem você mais pede ajuda?". "Familiares" ( $N=51$; $86,4 \%)$; "Colegas" ( $N=7 ; 11,9 \%)$ e "Docentes" ( $N=1 ; 1,7 \%)$, foram as principais categorias obtidas. Os participantes mencionaram pedir mais ajuda aos familiares. Poucos citaram pedir ajuda aos colegas e menos ainda aos docentes.

Saber a quem o aluno pode contar em momentos de dificuldades foi 0 assunto tratado na questão 7 que indagava "Em momentos difíceis,quem é que ajuda você?". Surgiram 5 categorias de respostas: "Mãe" (N=46; 44,6\%); "Pai" $(\mathrm{N}=28 ; 27,2 \%)$; "Demais Parentes" ( $N=14 ; 13,6 \%) ;$ "Irmão/ Irmã" ( $N=11$; $10,7 \%)$ e "Colegas" ( $N=4 ; 3,9 \%)$. A mãe a principal pessoa que os ajuda em um momento de necessidade, seguidamente do pai. Recorrer ao colega num momento de dificuldade foi pouco citado pelos participantes. É importante ressaltar que nesta questão não emergiu a categoria docente.

Respostas a questão 8: "Tem alguma matéria na escola que você mais pede ajuda? Qual? Porque?", revelaram que a maior parte dos alunos relatou pedir mais ajuda na disciplina de Matemática $(\mathrm{N}=19 ; 34,5 \%)$, seguida de Português $(N=16 ; 29,1 \%)$. Ciências $(N=10 ; 18,2 \%)$, História $(N=7 ; 12,7 \%)$, Geografia 
$(\mathrm{N}=2 ; 3,7 \%)$ e Educação Física $(\mathrm{N}=1 ; 1,8 \%)$, parecem envolver um menor uso dessa estratégia. A justificativa do pedir ajuda pelo estudante: "Impossibilidade de Realização Independente" ( $N=46 ; 88,5 \%)$ e "Desgostar da Matéria/ Estudo Insuficiente" ( $\mathrm{N}=6 ; 11,5 \%)$ foram as principais categorias.

A pergunta feita na questão 9 foi: "Você pede ajuda nas outras matérias? Em caso negativo porque não? Do total de 60 entrevistados, 20 estudantes disseram que não pediam ajuda em outra matéria, sendo que 7 (35\%) eram da $2^{a}$ série, $9(45 \%)$ da $3^{a}$ e $4(20 \%)$ da $4^{a}$ série. As categorias de respostas foram: "Saber Resolver" ( $\mathrm{N}=18 ; 90 \%)$ e "Preferência pelas Matérias" ( $\mathrm{N}=2$ ;10\%). A maior parte da amostra apresentou como principal justificativa não pedir ajuda nas demais matérias por conseguir realizar as tarefas.

A questão 10 tinha o objetivo de examinar a compreensão do aluno sobre o porquê ele foi ajudado em um momento de necessidade. A pergunta feita foi a seguinte: "Quando você precisa de ajuda e alguém lhe dá, você acha que essa pessoa the ajudou por quê?". Para esta questão, surgiram 6 categorias de repostas: "Qualidade Pessoal e Vínculos Afetivos" ( $\mathrm{N}=39 ; 65 \%)$; "Pela Simples Solicitação" ( $N=7 ; 11,7 \%)$; "Percepção da Dificuldade para a Realização Independente" (N=6; 10\%; ); "Interesses Particulares" $(\mathrm{N}=4 ; 6,7 \%)$; "Obrigação Moral ou Religiosa" ( $N=1 ; 1,6 \%)$ e 'Não Sei" ( $N=3 ; 5 \%)$. De modo geral, pode se constatar que os alunos relataram que foram ajudados devido à qualidade pessoal de quem ofereceu ajuda ou por laços afetivos existentes. Alguns participantes justificaram a ajuda recebida em função do pedido realizado. Outros alunos associaram a ajuda à dificuldade para fazer de algo de maneira independente ou tendo em vista interesses particulares.

Saber se o estudante ofereceria ajuda a alguém que estivesse precisando e porque foi o tópico abordado na última questão da entrevista. Todos os participantes disseram que ajudariam uma pessoa que estivesse necessitando. Emergiram sete categorias de repostas: "Retribuição da Ajuda" ( $N=23 ; 38,3 \%)$; "Qualidade Pessoal e Vínculos Afetivos" ( $\mathrm{N}=15 ; 25 \%)$; "Pela Necessidade" ( $\mathrm{N}=7$;

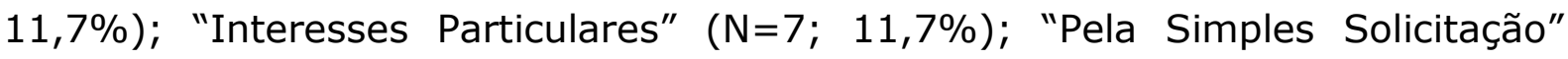
$(\mathrm{N}=3 ; 5 \%)$; "Obrigação Moral ou Religiosa" ( $\mathrm{N}=3 ; 5 \%)$; e "Não Sei" ( $\mathrm{N}=2$; $3,3 \%$ ). Retribuir alguma ajuda já recebida foi a principal justificativa dada pelos participantes. 


\section{DISCUSSÃO}

O objetivo do presente trabalho foi de conhecer as concepções sobre pedir ajuda de estudantes das séries iniciais do ensino fundamental. De modo geral, os estudantes relataram utilizar a estratégia de pedir ajuda, tanto para a realização de atividades escolares, como para resolver dificuldades que têm na sua vida cotidiana, principalmente quando não conseguem realizar algo de forma independente. A concepção do estudante acerca da estratégia de pedir ajuda dos alunos está voltada para um reconhecimento de dificuldades pessoais. Pedir ajuda se mostra, predominantemente, associado à dificuldade em realizar alguma atividade autonomamente, seja escolar ou não. A realização da tarefa escolar foi a situação em que os alunos revelaram pedir mais ajuda. Afirmaram, ainda, que o pedido de ajuda ocorre por meio da expressão verbal da dúvida ou do pedido de ajuda. Alguns participantes ressaltaram a importância da utilização de expressões socialmente valorizadas, tais como, Por favor e Obrigado(a).

A literatura da área indica também que a principal forma de expressar o pedido de ajuda é por meio da verbalização da dúvida. De acordo com Newman (1994), a decisão de pedir ajuda é realizada por meio de um sistema afetivomotivacional que envolve vários passos: primeiramente, o aluno deve ter consciência de que está com dificuldade para resolver a tarefa. O aluno reconhecer a dificuldade e buscar meios de realizar a tarefa é o que faz de pedir ajuda uma estratégia metacognitiva para a aprendizagem auto-regulada. Em seguida, deve avaliar todas as estratégias possíveis para solucionar a dificuldade e decidir pedir ajuda. Depois de ter tomado a decisão de pedir ajuda, deve escolher uma pessoa adequada para solicitá-la. Por último, o pedido de ajuda deve ser expresso de maneira apropriada, a fim de solucionar a dificuldade.

Os familiares são as principais pessoas a quem os estudantes relataram pedir ajuda. A mãe é a figura principal a quem eles mais procuram em situações de dificuldade, seguida do pai. Merece destaque o fato de o professor ter sido muito pouco mencionado como fonte de auxílio. Esse dado leva a um questionamento sobre o porquê estudantes procuram pouco ou não procuraram o professor para pedir ajuda, se passam boa parte do tempo sob a responsabilidade desse profissional? Foi interessante notar que tesultados semelhantes parecem ter ocorrido no ensino superior. No estudo realizado por Muneiro (2008), os estudantes universitários relataram pedir mais ajuda aos 
colegas do que ao professor, em especial os estudantes dos anos mais avançados.

De acordo com Butler (2006), a iniciativa de os estudantes pedirem ajuda ao professor depende essencialmente da forma como ele responde às dificuldades dos alunos e da forma como ele fornece. Dados de um estudo realizado por Turner et al (2002) revelaram que em salas de aula voltadas para a meta aprender é mais provável que não haja impedimentos para o pedir ajuda, já que os professores respondem mais às dificuldades dos estudantes, encorajando-os e oferecendo tempo e oportunidade para eles pensarem.

Constatou-se, no presente estudo, os vínculos afetivos, bem como a retribuição de uma ajuda e os valores religiosos e morais justificaram o receber e o dar ajuda. Para a estratégia de pedir ajuda a relação entre o ajudado e aquele que ajuda é fundamental, pois pedir ajuda se diferencia das outras estratégias de aprendizagem por envolver necessariamente outra pessoa (Newman, 2000, 2006).

\section{CONSIDERAÇÕES FINAIS}

De maneira geral, os dados da presente investigação indicam que os estudantes relatam utilizar a estratégia de pedir ajuda. A maior parte da amostra associou o pedido de ajuda ao reconhecimento de uma dificuldade em realizar alguma atividade por conta própria. Foi interessante observar que os alunos parecem pedir pouca ajuda aos professores, pois afirmaram pedir mais ajuda aos familiares e aos colegas (Serafim, 2009). Lamentavelmente, a ausência de estudos na literatura nacional abordando esse tema, torna difícil qualquer comparação ou discussão mais aprofundada dos dados.

Dada a relevância da estratégia de pedir ajuda para a aprendizagem, acredita-se que um ponto positivo deste trabalho foi seu caráter inovador, em termos da temática de estudo. A literatura internacional apresenta pesquisas sobre o pedir ajuda no contexto educacional, as quais presumivelmente se iniciaram por volta da década de 80 do século passado. No Brasil, essas investigações ainda são incipientes. Faz-se necessária a realização de outras pesquisas em nível nacional que explorem mais detalhadamente e aprofundem o conhecimento a respeito da estratégia de pedir ajuda em diferentes áreas, idades, séries e contextos educacionais. 
Como os instrumentos elaborados "Prancha Para Avaliação da Concepção de Pedir Ajuda de Alunos" e a "Entrevista - Conhecendo a Concepção de Pedir Ajuda de Alunos" se constituíram em uma primeira tentativa de medir e conhecer melhor o constructo pedir ajuda em nosso meio, espera-se também, também que eles sejam utilizados em futuras investigações, tendo em vista possíveis refinamentos.

\section{REFERÊNCIAS}

Bardin, L. (1991). Análise de conteúdo. Lisboa: Edições 70, LDA.

Boruchovitch, E. (1993). A psicologia cognitiva e a metacognição: Novas perspectivas para o fracasso escolar brasileiro. Tecnologia Educacional, 22(110/111), 22-28.

Boruchovitch, E. (1995). A identificação e o estudo das variáveis associadas ao fracasso escolar brasileiro. Projeto de pesquisa realizado na qualidade de bolsista de recém doutor da CNPQ. Faculdade de Educação, Departamento de Psicologia Educacional, UNICAMP.

Boruchovitch, E. (1998). Understanding brazilian students use of learning strategies. Resumo apresentado na 24th Internacional Congress of Applied Psycology, San Francisco, Agosto.

Boruchovitch, E. (1999). Estratégias de aprendizagem e desempenho escolar: Considerações para a prática educacional. Psicologia: Teoria e Crítica, 12(2), 391-376.

Boruchovitch, E. (2006). Estratégias de aprendizagem: Uma análise à luz das variáveis demográficas e motivacionais. Tese de Livre Docência. Faculdade de Educação - UNICAMP.

Costa, E. R. (2000). As estratégias de aprendizagem e ansiedade de alunos do ensino fundamental: Implicações para a prática educacional. Campinas, Dissertação de Mestrado, Faculdade de Educação, UNICAMP.

Butler, R. (2006). An achievement goal perspective on student help seeking and teacher help giving in the classroom: Theory, research, and educational implications. In S. A. Karabenick \& R. S. Newman (Eds.). Help seeking in academic settings. (pp. 45-88). Mahwah, NJ: Lawrence Erlbaum Associates.

Karabenick, S. A. (1998). Help seeking as a strategic resource. In S. A. Karabenick (Ed.). Strategic help seeking: implications for learning and teaching. (pp. 1-13). Hillsdale, NJ: Erlbaum. 
Karabenick, S. A., \& Knapp, J. R. (1991). Relationship of academic help seeking to the use of learning strategies and other instrumental achievement behavior in college students, Journal of Educational Psychology, 83(2), 221230.

Marchand, G., \& Skinner, E. A. (2007). Motivational dynamics of children's academic help-seeking and concealment. Journal of Educational Psychology, 99(1), 65-82.

Muneiro, M. L. (2008). Estratégias de aprendizagem de alunos do ensino superior: Cursos de serviço social e engenharia de produção elétrica. Dissertação de Mestrado, Faculdade de Educação, Universidade Estadual de Campinas, Campinas.

Newman, R. S. (1990). Children's help seeking in the classroom; the role of motivational factors and attitudes. Journal of Educational Psychology, 83(1), 71-80.

Newman, R. S. (1991). Goals and self-regulated learning: What motivates children to seek academic help? In M. L. Maehr \& P. R. Pintrich (Eds.). Advances in motivation and achievement. (pp. 151-183). Greenwich, CT: JAI.

Newman, R.S. (1994) Adaptive help seeking: a strategy of self-regulated learning. In D. H. Schunk \& B. J. Zimmerman (Eds.). Self-regulation of learning and performance: issues and educational applications. (pp. 282301). Hillsdale, NJ: Lawrence Erlbaum Associates.

Newman, R.S. (1998) Adaptive help-seeking: a role of social interaction in selfregulated learning. In S. A. Karabenick (Ed.). Strategic help seeking: implications for learning and teaching. (pp. 13-37). Hillsdale, NJ: Erlbaum.

Newman, R. S. (2000). Social influences on the development of children's adaptive help seeking: the role of parents, teachers, and peers. Developmental Review, 20(3), 350-404.

Newman, R. S. (2002). How self-regulated learners cope with academic difficulty: The role of adaptative help seeking. Theory into Practice, 41(2), $132-138$

Newman, R. S. (2006). Students' adaptive and nonadptive help seeking in the classroom: Implications for the context of peer harassment. In $\mathrm{S}$. Karabenick \& R. S. Newman (Eds.). Help seeking in academic settings: goals, groups, and contexts. (pp. 225-258). Lawrence Erlbaum Associates.

170 Estudos Interdisciplinares em Psicologia, Londrina, v. 1, n. 2, p. 159-171, dez. 2010 
Ryan, A. M., \& Pintrich, P. R. (1997). "Should I ask for help?" The role of motivation and attitudes in adolescents' help seeking in math class. Journal of Educational Psychology, 89(2), 329-341.

Schlieper, M. D. M. J. (2001). As estratégias de aprendizagem e as atribuições de causalidade de alunos do ensino fundamental. Dissertação de Mestrado, Faculdade de Educação, Universidade Estadual de Campinas, Campinas

Serafim, T. M. (2004). As estratégias de aprendizagem dos alunos do ensino fundamental: uma análise por gênero, série escolar e idade. Trabalho de Conclusão de Curso, Faculdade de Educação, Universidade Estadual de Campinas, Campinas.

Serafim, T. M. (2009). A estratégia de pedir ajuda de estudantes do ensino fundamental. Dissertação de Mestrado, Faculdade de Educação, Universidade Estadual de Campinas, Campinas.

Serafim \& Boruchovitch (2007). Escala de avaliação da estratégia de pedir ajuda no contexto escolar. Manuscrito não publicado. Universidade Estadual de Campinas, Campinas.

Turner, J. C., Midgley, C., Meyer, D. K., Gheen, M. Andertman, E. M., Kang, Y. \& Patrick, H. (2002). The classroom environment and students' reports of avoidance strategies in mathematics: A multi-method study. Journal of Educational Psychology, 94(1), 88-106.

Van Der Meij, H. (1988). Constrains on question asking in classrooms. Journal of Psychology Educational, 80(3), 401-405.

Van Der Meij, H. (1990). Question asking: to know that you do not know is not enough. Journal of Psychology Educational, 82(3), 505-512.

Contato: taniaserafim@yahoo.com, evely@unicamp.br

Recebido em: 29/09/2010

Revisado em: 10/11/2010

Aceito em: 06/12/2010 